\title{
Lip Morphological Changes and Differences before and after Orthodontic Treatment
}

\author{
Islam MR ${ }^{1}$, Kitahara $T^{2}$, Naher $\mathbf{L}^{3}$, Nakasima $\mathrm{A}^{4}$ and Takahashi $\mathbf{I}^{5}$
}

\begin{abstract}
Objectives: The objective of this study is to test the hypothesis that there is a difference in the morphology of the lips of Class II div 1 and Class III malocclusion, before and after orthodontic treatment.

Methods: The sample subjects included 20 adult female patients with Angle Class II div 1 malocclusion and 30 adult female patients with dento-skeletal Class III malocclusion. Frontal photographs were taken both before and after orthodontic and/or orthognathic treatment. Thirty-five landmarks were placed on each tracing made from the photograph. Thereafter, landmarks were digitized into an $\mathrm{x}$ and $\mathrm{y}$ coordinates system with the subnasal point as the origin. The Class II pretreatment and posttreatment groups of rest and smile conditions were compared with the Class III group respectively, using t-tests.
\end{abstract}

Results: In the Class II pretreatment group, upper and lower lips positioned downward than that in the Class III group. When smiling, there was no statistically significant difference between the two groups before treatment, whereas after orthodontic treatment both Class II and Class III groups showed the significant difference at rest and on smiling conditions. On smiling condition Class III group showed more upwards and lateral movement of the mouth corners than Class II group.

Conclusion: Orthodontic treatment induced larger amount in movement of lips at smiling in both Class II and III patients, which may reflect the emotional changes in the patients after treatment.

KEY WORDS: Smile; Soft tissue; Class II Malocclusion; Class III Malocclusion; Orthognathic surgery. (Ban J Orthod \& Dentofac Orthop, October 2010; Vol-1, No. 1, 5-12)

\section{INTRODUCTION}

The treatment goal ${ }^{1-2}$ of modern orthodontic and orthognathic treatment is to produce morphological and functional harmony in maxillofacial complex. Orthodontic researches had evaluated the structural harmony in craniofacial skeletons from early to middle 20th century, and many different types of the cephalometric analyses had been established based on the hard tissue morphology of the patients on the cephalometric radiographs. While most of those parameters were utilized on the lateral cephalometric analysis at resting or enforced lip closing positions, those concepts lacked to estimate the frontal appearance of the face and dynamic relationship between tooth and lips during they are functioning. Consequently, in addition to static evaluation of the frontal balance of the face, smile attractiveness was considered to be critical parameter to evaluate the orthodontic outcomes. Smile arc and tooth exposure on smile are the common parameter to evaluate the dynamic function of the lips. It was demonstrated that the attractiveness of smile is enhanced when smile arc of lower lip coincides with the tip lines of upper anterior teeth, and the anterior tooth exposure is about 75 to $100 \%$ while smiling. The current interest in smile enhancement is overdue and smile esthetics has become increasingly important in modern orthodontics. The smile is an important form of facial expression. Facial expression and physical attractiveness in general form essential parts of social interaction. A pleasing smile clearly enhance of an individual in our society. On the other hand, a defective smile might be considered properly as a physical handicap. It has been well documented that considerable importance is placed on dental aesthetics, and both parents and their children feel that pleasant dental aesthetics are an important factor for physical well being. Persons with crowded dentitions are not only considered less attractive by lay people, but also are perceived to be less intelligent, whereas children with well aligned teeth are thought to be friendlier, of a higher social class, more popular and more intelligent. Young adults are aware, to varying extents, of anterior occlusal traits and this is particularly so in those who seek orthodontic treatment.

The improvement of facial esthetics has rapidly become one of the desirable objectives of orthodontic treatment. The objective of orthodontic treatment is to achieve a harmonious relationship between the skeletal, dental and soft tissue for the improvement of the function and facial esthetics. If an orthodontist is to adapt

\footnotetext{
${ }^{1}$ Assistant Professor, Department of Orthodontics, Pioneer Dental College, Dhaka University, Bangladesh. ${ }^{2}$ Assistant Professor, Department of Orthodontics, Graduate School of Dentistry, Kyushu University, Fukuoka, Japan ${ }^{3}$ Assistant Professor, Department of Oral Pathology and Medicine, Pioneer Dental College, Dhaka University, Bangladesh. ${ }^{4}$ Emeritus Professor, Department of Orthodontics, Graduate School of Dentistry, Kyushu University, Fukuoka, Japan. ${ }^{5}$ Professor \& Chairman, Department of Orthodontics, Graduate School of Dentistry, Kyushu University, Fukuoka, Japan.
} 
his treatment to modifying facial appearance, it would seem important to know what change will usually occur within the soft tissues after orthodontic treatment. Zachrisson ${ }^{3}$ stated that clinical assessment prior to orthodontic treatment should always include an evaluation of the soft tissues at rest and during function. Wylie 4 stated " the goal of orthodontic treatment should be the attainment of the best possible esthetic result, dentally and facially." At present, orthodontic patients are concerned with their dynamic appearances during conversation and smiling, in addition to their static appearances. Roy Sabri ${ }^{5}$ also stated that patients come to orthodontist mainly to improve their smiles.

Therefore, a statistical evaluation of the morphological changes in the lips and soft tissues of the perioral region after orthognathic treatment was conducted.

The orthodontic literature contains more studies on skeletal structure, and the smile still receives relatively little attention. Most of the reports on the dentofacial changes have been based on cephalometric data. However, in cephalogram the soft tissue structures are only in profile and limited to the anterior most outlines (Samir E. Bishara6, 1995). Lateral cephalograms and silhouettes have the advantage of reducing or eliminating the influence of confounding variables (Foster ${ }^{7}$, 1973) but they do not represent the whole face and the actual smile cannot be evaluated (Mackley8, 1993). Frontal photographs generally are rated more attractive than profile views (Kerr and O'Donnell ${ }^{9}$, 1990). The advantage of this facial photograph based study is that the procedure is simple, economical and easy to increase the number of samples.
In our study, we used posed smile. The posed smile is static in the sense that it can be sustained. The lip animation is fairly reproducible (David M. Sarver ${ }^{10}$, 2001).

The objective of this study is to test the hypothesis that there is a different in the morphology of the lips of Class II div 1 and Class III malocclusion at rest and on smiling conditions, before and after orthodontic treatment.

\section{MATERIALS AND METHODS}

The sample subjects included 20 adult female patients (age range 18-35 years; mean 22.2 years) with Angle Class II division 1 malocclusion and a mean over jet of $7.4 \mathrm{~mm}$ and over bite of 3.8 $\mathrm{mm}$ and 30 adult female Angle Class III patients (age range 1832 years; mean $23.8 \pm 4.7$ years) with mandibular prognathism who underwent an orthognathic surgical treatment. The surgical treatments were performed with either SSRO (17 patients) or IVRO (13 patients) without genioplasty surgery, twenty-five subjects were treated with tooth extraction and five subjects were treated without extraction (Table 1). All of the patients were treated at the Kyushu University Hospital, Orthodontic Clinic from 2001 to 2007. This study was carried out in accordance with the regulations of the Ethical Committee of the Faculty of Dentistry of Kyushu University, and informed consent was obtained from each subject prior to data collection.

Table 1. Summary of Class-II \& Class-III patients

\begin{tabular}{|c|c|c|c|c|}
\hline & & $\begin{array}{l}\text { Class II group } \\
\qquad(\mathrm{N}=20) \\
\text { Mean }+\mathrm{SD}\end{array}$ & \multirow{2}{*}{\multicolumn{2}{|c|}{$\begin{array}{l}\text { Class III group } \\
\qquad \begin{array}{c}(\mathrm{N}=30) \\
\text { Mean } \pm \mathrm{SD}\end{array}\end{array}$}} \\
\hline & & Mean \pm SD & & \\
\hline Age of the patients & Yrs. & $22.2 \pm 5.6$ & \multicolumn{2}{|c|}{$23.8 \pm 4.7$} \\
\hline Treatment period & Yrs. & $2.3 \pm 0.5$ & \multicolumn{2}{|c|}{$2.7 \pm 0.8$} \\
\hline Tooth extracted & Number & Ext $=17$ Non-Ext $=3$ & $\mathrm{Ext}=25$ & Non-Ext $=5$ \\
\hline Opreration & Number & - & $\mathrm{SSRO}=17$ & IVRO=13 \\
\hline
\end{tabular}

The photographic procedure was described previously11. The frontal photographs of the patients were taken at the two stages of the start of the pre-surgical orthodontic treatment and the immediately after post-surgical treatment in a normal seated posture with the head fixed by ear rods, at a distance of $1.5 \mathrm{~m}$ between the camera lens and the subject at rest and posed smiling condition. The subjects wore no facial cosmetics/makeup. The subject's head was positioned so that the Frankfort horizontal plane was parallel to the floor, and the mid sagittal plane of the head was aligned with the center of the camera lens. The criteria for inclusion in the study were the availability of a standardized facial photograph of adequate quality and resolution, taken according to a strict data collection protocol. Each subject was coached and asked to achieve the same lip position at least twice in succession before a photograph was taken. While posed smile, they kept their molars lightly close, and the perioral soft tissues and mandibular posture were unstrained at rest. The frontal photographs were printed on A4 size paper, and tracings were made and 35 facial landmarks were added using tracing paper (Figure 1). This study fixed the subnasal (Sn) point as the origin. A line was drawn through the center of the eyeball. A horizontal plane was drawn through the Sn point parallel to the eyeball distance line, and this plane was designated as the x-axis. A vertical line was drawn perpendicular to the $x$-axis through the Sn point, which was 

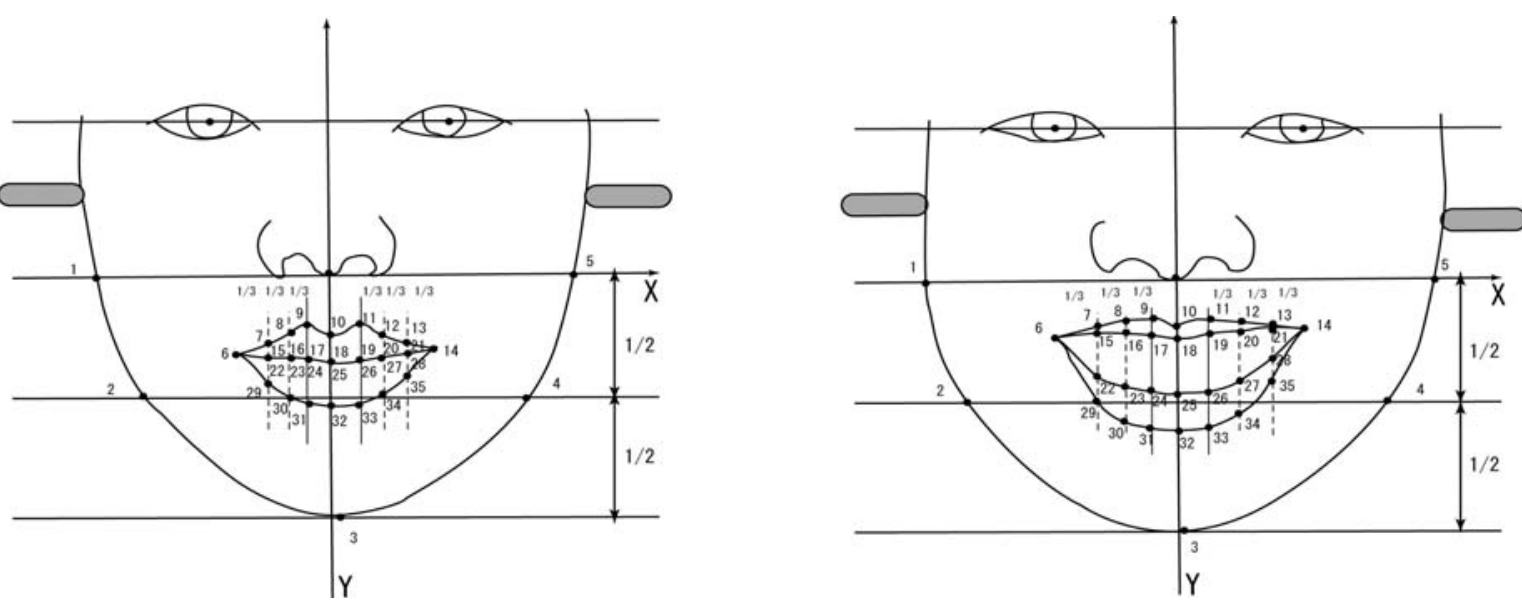

Figure 1. Facial landmarks. (1) Zygion (right). (3) Soft tissue Pogonion. (5) Zygion (left). (6) Commissure (right). (9) Christa Philtri (right). (10)Vermillion superior. (11) Christa Philtri (left). (14) Commissure (left). (32) Vermillion inferior. *6-14, 15-21. Upper lip. *22-28, 29-35. Lower lip.

designated as the y-axis. Next, another line was drawn parallel to the $\mathrm{x}$-axis through the lower border of the chin, and the $\mathrm{x}$-axis to the lower border of the chin was divided into two equal halves. Then, two vertical lines were drawn through the right and left superior vermilion point $(9,11)$. From the superior vermilion point of the lip to the corners of the mouth both the right (6) and left (14) sides were divided into three equal parts. Every landmark was digitized into $\mathrm{x}$ - and $\mathrm{y}$-coordinate values, and a statistical analysis was performed using these values. The landmarks numbered 6-14 and 15-21 indicated the upper lip area, and 22- 28 and 29-35 indicated the lower lip area. We examined the differ- ences in the facial size by measuring the distance between the center of the right and left eyeballs of the Class II and Class III groups. There were no statistically significant differences between the two groups with different facial patterns (Table 2). The pretreatment rest and smile conditions were compared with the posttreatment conditions, of Class II and the Class III groups were compared by using two sample t-test using the Microsoft Excel software program (Microsoft Corporation, Redmond, Wash). Differences with a value of $\mathrm{P}<.05$ were considered to be statistically significant.

Table 2. Measurement of Facial Size

\begin{tabular}{lccc}
\hline Point & Class II patient group & Class III patient group & \\
$\mathrm{mm}$ & Mean \pm SD & Mean \pm SD & "Student`s t-test" \\
\hline Po-Or & $73.6 \pm 4.08$ & $74.55 \pm 2.99$ & NS \\
\hline
\end{tabular}

* $\mathrm{P}<0.05 ; * * \mathrm{P}<0.01 ; * * * \mathrm{P}<0.001$. NS $=$ Not Significant

Po-Or indicates the average distance between the two eye balls.

\section{Error of Methods}

The systematic and accidental errors of analysis were evaluated by duplicate determinations of 25 photographs selected at random. Selected photographs were retraced and recalculated by the same person about one month after the initial data was recorded. The error variance was calculated according to the Dahlberg formula and systematic error between the first and second measurements was calculated using the paired t-test. Most of the acci- dental errors smaller than $1 \mathrm{~mm}$ and the errors did not exceed $0.59 \mathrm{~mm}$. In addition, the coefficients of reliability values were high, thus indicating the sufficient accuracy of the measurements. (Table 3)

The same facial photograph traced 30 times to evaluate the intraexaminer error (Table 4). The mean of the error in the $\mathrm{x}$ and $\mathrm{y}$ coordinate values, expressed by the coefficient of variation, was .05 and .01 , respectively. 
Table 3. Error of the Merthod Assessed From Duplicate Tracings of 25 Photographs.

\begin{tabular}{|c|c|c|c|c|c|c|c|}
\hline Point & Sl No. & $\begin{array}{l}\text { Dahlberg's } \\
\text { Calculation }\end{array}$ & $\begin{array}{l}\text { Houston's } \\
\text { Coefficient of } \\
\text { Reliability }\end{array}$ & $\begin{array}{l}\text { Systematic } \\
\text { Error: t-test } \\
\text { (P Value) }\end{array}$ & $\begin{array}{l}\text { Dahlberg's } \\
\text { Calculation }\end{array}$ & $\begin{array}{l}\text { Houston's } \\
\text { Coefficient of } \\
\text { Reliability }\end{array}$ & $\begin{array}{l}\text { Systematic } \\
\text { Error: t-test } \\
\text { (P Value) }\end{array}$ \\
\hline \multirow[t]{5}{*}{ Outline } & 1 & 0.485 & 0.995 & $0.00005^{*}$ & - & - & - \\
\hline & 2 & 0.499 & 0.994 & 0.660 & 0.492 & 0.962 & 0.284 \\
\hline & 3 & - & - & - & 0.504 & 0.991 & 0.270 \\
\hline & 4 & 0.594 & 0.994 & $0.004 *$ & 0.496 & 0.966 & 0.185 \\
\hline & 5 & 0.448 & 0.987 & 0.927 & - & - & - \\
\hline \multirow[t]{16}{*}{ Upper lip } & 6 & 0.356 & 0.991 & 0.311 & 0.396 & 0.995 & 0.118 \\
\hline & 7 & 0.349 & 0.987 & 0.302 & 0.459 & 0.989 & 0.811 \\
\hline & 8 & 0.263 & 0.993 & 0.876 & 0.448 & 0.983 & 0.580 \\
\hline & 9 & 0.286 & 0.994 & 0.810 & 0.564 & 0.974 & 0.104 \\
\hline & 10 & - & - & - & 0.458 & 0.981 & 0.451 \\
\hline & 11 & 0.365 & 0.993 & 0.202 & 0.456 & 0.984 & 0.057 \\
\hline & 12 & 0.398 & 0.988 & 0.918 & 0.522 & 0.979 & 0.854 \\
\hline & 13 & 0.369 & 0.989 & 0.213 & 0.462 & 0.991 & $0.014^{*}$ \\
\hline & 14 & 0.193 & 0.998 & 0.355 & 0.488 & 0.994 & 0.581 \\
\hline & 15 & 0.305 & 0.990 & 0.928 & 0.435 & 0.993 & 0.188 \\
\hline & 16 & 0.247 & 0.994 & 0.616 & 0.435 & 0.990 & 0.211 \\
\hline & 17 & 0.294 & 0.993 & 0.744 & 0.414 & 0.989 & 0.127 \\
\hline & 18 & - & - & - & 0.358 & 0.991 & 0.116 \\
\hline & 19 & 0.286 & 0.996 & 0.128 & 0.489 & 0.987 & $0.021^{*}$ \\
\hline & 20 & 0.378 & 0.989 & 0.585 & 0.410 & 0.992 & $0.036^{*}$ \\
\hline & 21 & 0.335 & 0.990 & 0.712 & 0.395 & 0.994 & 0.108 \\
\hline \multirow[t]{14}{*}{ Lower lip } & 22 & 0.278 & 0.992 & 0.083 & 0.480 & 0.994 & 0.136 \\
\hline & 23 & 0.257 & 0.994 & 0.957 & 0.311 & 0.997 & 0.627 \\
\hline & 24 & 0.223 & 0.996 & 0.666 & 0.409 & 0.994 & 0.065 \\
\hline & 25 & - & - & - & 0.358 & 0.995 & 0.212 \\
\hline & 26 & 0.361 & 0.994 & 0.077 & 0.460 & 0.995 & 0.953 \\
\hline & 27 & 0.384 & 0.989 & 0.694 & 0.565 & 0.990 & 0.526 \\
\hline & 28 & 0.305 & 0.992 & 0.364 & 0.460 & 0.995 & 1.000 \\
\hline & 29 & 0.280 & 0.992 & 0.961 & 0.597 & 0.992 & 0.963 \\
\hline & 30 & 0.315 & 0.990 & 0.271 & 0.484 & 0.994 & 0.116 \\
\hline & 31 & 0.234 & 0.996 & 0.443 & 0.390 & 0.995 & 0.140 \\
\hline & 32 & - & - & - & 0.495 & 0.992 & $0.007^{*}$ \\
\hline & 33 & 0.414 & 0.995 & $0.0004^{*}$ & 0.417 & 0.994 & 0.138 \\
\hline & 34 & 0.442 & 0.985 & 0.533 & 0.532 & 0.993 & 0.239 \\
\hline & 35 & 0.392 & 0.987 & 0.305 & 0.528 & 0.994 & 0.247 \\
\hline
\end{tabular}

$* \mathrm{P}<0.05$

\section{RESULTS}

Table 5 shows there is no significant difference of the lips area between class II and class III groups at rest condition, The upper and lower lip ratio (U/L ratio) was $82 \%$ of class II group and $80 \%$ of class III group. On smiling condition, upper lip area of class III group showed larger than class II group $(\mathrm{P}<.05)$. The class II pretreatment group in rest condition, both lips and mouth corners positioned downward than that of class III group. When smiling there was no statistically significant difference between two groups, except facial out line region (Table 6 and Figure 2).

The coordinates of the class III pretreatment group in rest and smiling conditions, clear significant $(\mathrm{P}<.05)$ differences in vertical axis, where facial outline placed to an inferior position. This is due to mandibular prognathism (Table 6 and Figure 2).
After treatment the result shows (Table 5) that, in the both rest and smiling conditions upper lip area of class III group was larger than class II group, as well as upper and lower lip ratios were significantly difference. The lip ratio (U/L ratio) was $65 \%$ at of class II group and $70 \%$ of class III group. Lower lip balance by the orthognathic treatment.

After orthodontic treatment, both class II and class III groups showed a significant difference at rest and smiling conditions. Specially on smiling condition class III group showed more upwards and lateral $(\mathrm{P}<.05, \mathrm{P}<.01, \mathrm{P}<.001)$ movement of the mouth corners than class II group. In the both rest and smile conditions, posttreatment group showed there is no significant difference due to mandible became shorter after orthognathic treatment of class III group inferior to those of control (Table 7 and Figure 3). 
Lip Morphological Changes and Differences before and after Orthodontic Treatment.

Table 4. Intra examiner error

\begin{tabular}{|c|c|c|c|c|c|c|c|c|c|c|c|c|c|}
\hline \multicolumn{4}{|c|}{$\mathrm{X}$} & \multicolumn{3}{|c|}{$\mathrm{Y}$} & \multicolumn{4}{|c|}{$\mathrm{X}$} & \multicolumn{3}{|c|}{$\mathrm{Y}$} \\
\hline $\begin{array}{l}\text { Land- } \\
\text { marks }\end{array}$ & Mean & SD & $\begin{array}{l}\text { Standard } \\
\text { Error }\end{array}$ & Mean & SD & $\begin{array}{l}\text { Standard } \\
\text { Error }\end{array}$ & $\begin{array}{l}\text { Land- } \\
\text { marks }\end{array}$ & Mean & SD & $\begin{array}{l}\text { Standard } \\
\text { Error }\end{array}$ & Mean & SD & $\begin{array}{l}\text { Standard } \\
\text { Error }\end{array}$ \\
\hline $\mathrm{S}$ & - & - & - & - & - & - & 17 & -5.1 & 0.27 & 0.05 & -9.1 & 0.17 & 0.03 \\
\hline $\mathrm{N}$ & - & - & - & 33.4 & 0.19 & 0.04 & 18 & - & - & - & -9.4 & 0.17 & 0.03 \\
\hline Or & 20.2 & 0.22 & 0.04 & 33.4 & 0.15 & 0.03 & 19 & 5.0 & 0.25 & 0.05 & -9.4 & 0.18 & 0.03 \\
\hline Po & -19.4 & 0.19 & 0.03 & 33.4 & 0.15 & 0.03 & 20 & 10.0 & 0.24 & 0.04 & -10.1 & 0.22 & 0.04 \\
\hline 1 & -42.9 & 0.17 & 0.03 & 0.0 & 0.00 & 0.00 & 21 & 14.9 & 0.26 & 0.05 & -10.7 & 0.16 & 0.03 \\
\hline 2 & -35.2 & 0.19 & 0.04 & -23.5 & 0.29 & 0.05 & 22 & -15.1 & 0.26 & 0.05 & -14.4 & 0.28 & 0.05 \\
\hline 3 & 0.3 & 0.33 & 0.06 & -47.5 & 0.20 & 0.04 & 23 & -10.2 & 0.22 & 0.04 & -18.7 & 0.19 & 0.04 \\
\hline 4 & 38.7 & 0.22 & 0.04 & -23.2 & 0.17 & 0.03 & 24 & -5.2 & 0.19 & 0.03 & -20.3 & 0.16 & 0.03 \\
\hline 5 & 45.2 & 0.19 & 0.04 & - & - & - & 25 & - & - & - & -20.7 & 0.18 & 0.03 \\
\hline 6 & -19.0 & 0.22 & 0.04 & -9.8 & 0.16 & 0.03 & 26 & 5.0 & 0.20 & 0.04 & -20.7 & 0.23 & 0.04 \\
\hline 7 & -15.3 & 0.17 & 0.03 & -7.9 & 0.21 & 0.04 & 27 & 9.9 & 0.19 & 0.03 & -19.6 & 0.25 & 0.05 \\
\hline 8 & -10.3 & 0.17 & 0.03 & -7.0 & 0.21 & 0.04 & 28 & 14.6 & 0.21 & 0.04 & -16.4 & 0.15 & 0.03 \\
\hline 9 & -5.3 & 0.20 & 0.04 & -6.4 & 0.13 & 0.02 & 29 & -15.1 & 0.17 & 0.03 & -18.9 & 0.22 & 0.04 \\
\hline 10 & - & - & - & -7.2 & 0.20 & 0.04 & 30 & -10.3 & 0.17 & 0.03 & -23.8 & 0.21 & 0.04 \\
\hline 11 & 4.8 & 0.14 & 0.02 & -6.8 & 0.22 & 0.04 & 31 & -5.0 & 0.27 & 0.05 & -26.1 & 0.19 & 0.04 \\
\hline 12 & 9.9 & 0.14 & 0.03 & -7.4 & 0.23 & 0.04 & 32 & - & - & - & -26.9 & 0.19 & 0.03 \\
\hline 13 & 14.9 & 0.25 & 0.05 & -9.0 & 0.18 & 0.03 & 33 & 5.0 & 0.24 & 0.04 & -26.6 & 0.21 & 0.04 \\
\hline 14 & 19.0 & 0.24 & 0.04 & -10.9 & 0.17 & 0.03 & 34 & 9.8 & 0.23 & 0.04 & -24.7 & 0.17 & 0.03 \\
\hline 15 & -15.1 & 0.24 & 0.04 & -9.4 & 0.21 & 0.04 & 35 & 14.6 & 0.19 & 0.03 & -20.4 & 0.19 & 0.03 \\
\hline 16 & -10.2 & 0.21 & 0.04 & -9.3 & 0.20 & 0.04 & & & & & & & \\
\hline
\end{tabular}

Table 5. Area measurements.Comparison bet Class-II and Class-III

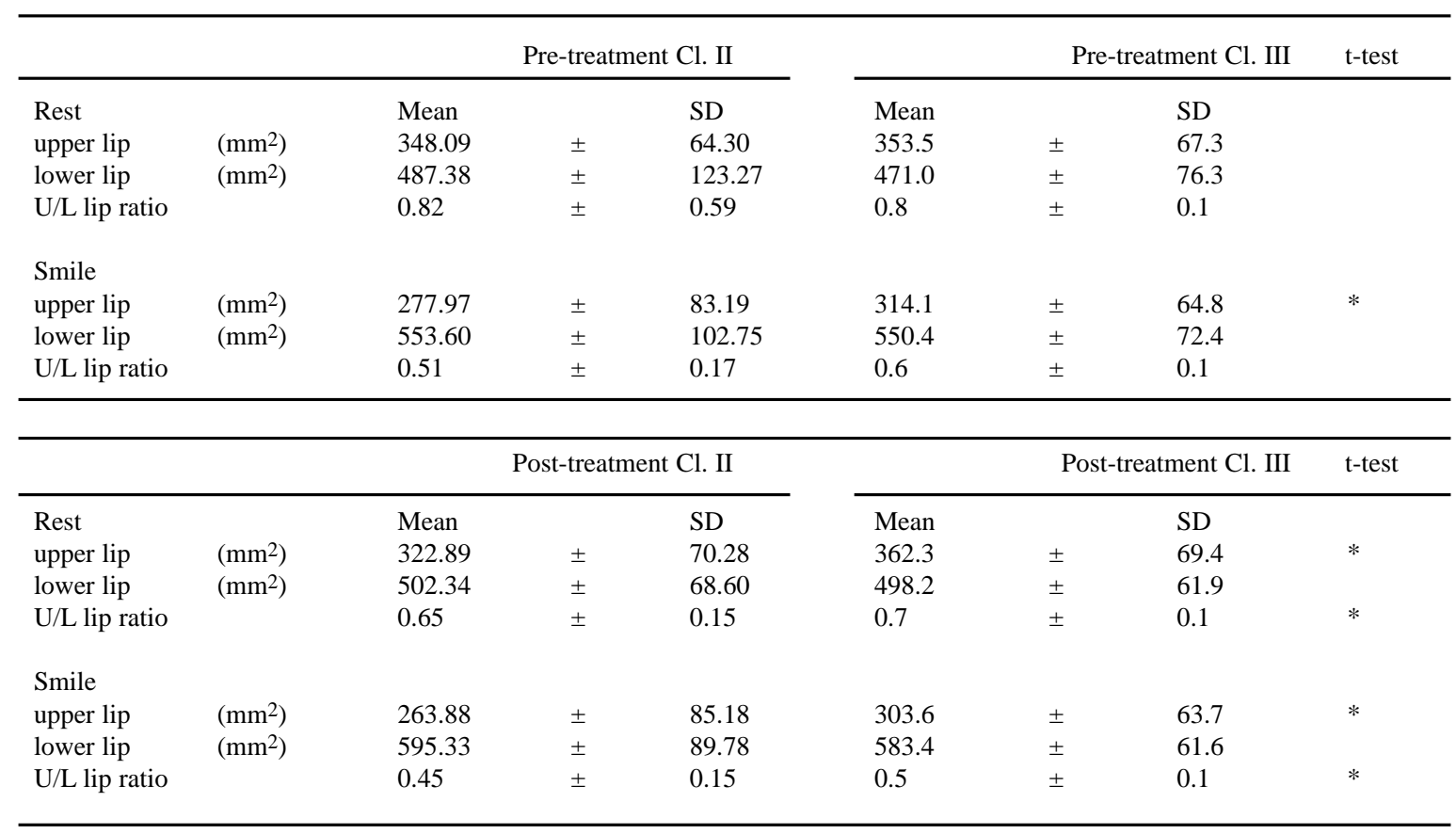

Bangladesh Journal of Orthodontics and Dentofacial Orthopedics (BJO \& DFO) 
Islam MR, Kitahara T, Naher L, Hossain MZ, Nakasima A \& Takahashi I

Table 6. Comparism between the Class II pretreatment group from the Class III pretreatment group

\begin{tabular}{|c|c|c|c|c|c|c|c|c|c|c|c|c|c|c|c|c|c|}
\hline \multirow[b]{3}{*}{ Point } & & \multicolumn{8}{|c|}{ Pre Rest } & \multicolumn{8}{|c|}{ Pre Smile } \\
\hline & & \multicolumn{4}{|c|}{$\mathrm{X}$} & \multicolumn{4}{|c|}{$\mathrm{Y}$} & \multicolumn{4}{|c|}{$X$} & \multicolumn{4}{|c|}{$\mathrm{Y}$} \\
\hline & & $\begin{array}{l}\text { ClassII } \\
\text { Mean }\end{array}$ & \pm & \multicolumn{2}{|c|}{$\begin{array}{l}\text { ClassIII } \\
\text { Mean }\end{array}$} & \multirow{2}{*}{$\begin{array}{l}\text { ClassII } \\
\text { Mean } \\
0.0\end{array}$} & \pm & \multicolumn{2}{|c|}{$\begin{array}{l}\text { ClassIII } \\
\text { Mean }\end{array}$} & \multirow{2}{*}{$\begin{array}{l}\begin{array}{l}\text { ClassII } \\
\text { Mean }\end{array} \\
-78.6\end{array}$} & \pm & \multicolumn{2}{|c|}{$\begin{array}{l}\text { ClassIII } \\
\text { Mean }\end{array}$} & \multirow{2}{*}{$\begin{array}{l}\begin{array}{l}\text { ClassII } \\
\text { Mean }\end{array} \\
0.0\end{array}$} & & \multicolumn{2}{|c|}{$\begin{array}{l}\text { ClassIII } \\
\text { Mean }\end{array}$} \\
\hline Outline, mm & 1 & -77.4 & \pm & -77.7 & & & \pm & 0.2 & & & \pm & -79.4 & & & \pm & 0.2 & \\
\hline & 2 & -62.0 & \pm & -61.8 & & -38.0 & \pm & -39.8 & * & -62.1 & \pm & -63.1 & & -39.0 & \pm & -41.3 & $* *$ \\
\hline & 3 & 0.0 & \pm & 0.0 & & -76.1 & \pm & -79.8 & * & -1.1 & \pm & 0.0 & $* *$ & -78.3 & \pm & -82.4 & $* *$ \\
\hline & 4 & 60.5 & \pm & 62.2 & & -38.4 & \pm & -40.1 & * & 60.2 & \pm & 62.9 & $*$ & -39.3 & \pm & -41.4 & $* *$ \\
\hline & 5 & 75.6 & \pm & 78.2 & * & 0.0 & \pm & -0.1 & & 76.1 & \pm & 79.1 & $* *$ & 0.0 & \pm & -0.1 & \\
\hline \multirow[t]{16}{*}{ Upper lip, mm } & 6 & -28.6 & \pm & -28.1 & & -27.6 & \pm & -24.8 & $* * *$ & -34.1 & \pm & -33.5 & & -20.1 & \pm & -19.4 & \\
\hline & 7 & -21.7 & \pm & -21.0 & & -23.3 & \pm & -20.0 & $* * *$ & -26.2 & \pm & -25.5 & & -16.4 & \pm & -15.3 & \\
\hline & 8 & -14.7 & \pm & -13.9 & & -19.3 & \pm & -16.3 & $* * *$ & -17.7 & \pm & -18.0 & & -14.3 & \pm & -12.7 & $*$ \\
\hline & 9 & -7.4 & \pm & -6.5 & $*$ & -16.9 & \pm & -14.0 & $* * *$ & -9.3 & \pm & -10.2 & & -13.1 & \pm & -11.1 & $*$ \\
\hline & 10 & 0.0 & \pm & 0.0 & & -18.5 & \pm & -15.3 & $* * *$ & 0.0 & \pm & 0.0 & & -14.4 & \pm & -12.1 & $* *$ \\
\hline & 11 & 7.0 & \pm & 7.4 & & -16.8 & \pm & -13.9 & $* * *$ & 9.3 & \pm & 11.5 & $*$ & -13.0 & \pm & -11.0 & $*$ \\
\hline & 12 & 14.4 & \pm & 14.3 & & -19.1 & \pm & -16.2 & $* * *$ & 17.2 & \pm & 18.7 & & -14.3 & \pm & -12.6 & \\
\hline & 13 & 21.7 & \pm & 21.6 & & -22.8 & \pm & -20.2 & $* * *$ & 25.0 & \pm & 25.9 & & -16.5 & \pm & -15.2 & \\
\hline & 14 & 28.3 & \pm & 28.7 & & -26.9 & \pm & -24.5 & $* *$ & 32.3 & \pm & 33.5 & & -19.6 & \pm & -19.2 & \\
\hline & 15 & -21.7 & \pm & -21.0 & & -27.4 & \pm & -24.4 & $* * *$ & -26.2 & \pm & -25.5 & & -19.3 & \pm & -18.9 & \\
\hline & 16 & -14.7 & \pm & -13.8 & & -26.8 & \pm & -24.0 & $* * *$ & -17.7 & \pm & -18.0 & & -19.1 & \pm & -18.2 & \\
\hline & 17 & -7.5 & \pm & -6.6 & * & -26.5 & \pm & -23.8 & $* *$ & -9.3 & \pm & -10.2 & & -19.2 & \pm & -17.8 & \\
\hline & 18 & 0.0 & \pm & 0.0 & & -26.9 & \pm & -24.1 & $* * *$ & 0.0 & \pm & 0.0 & & -20.4 & \pm & -18.3 & \\
\hline & 19 & 7.0 & \pm & 7.5 & & -26.5 & \pm & -24.0 & $* *$ & 9.3 & \pm & 11.5 & $*$ & -19.5 & \pm & -17.8 & \\
\hline & 20 & 14.4 & \pm & 14.3 & & -26.5 & \pm & -24.0 & $* *$ & 17.1 & \pm & 18.7 & & -19.3 & \pm & -18.3 & \\
\hline & 21 & 21.7 & \pm & 21.6 & & -26.8 & \pm & -24.7 & $* *$ & 24.9 & \pm & 25.9 & & -19.3 & \pm & -19.0 & \\
\hline \multirow[t]{14}{*}{ Lower lip, mm } & 22 & -21.8 & \pm & -21.0 & & -27.5 & \pm & -24.3 & $* * *$ & -26.2 & \pm & -25.5 & & -25.5 & \pm & -25.4 & \\
\hline & 23 & -14.7 & \pm & -13.8 & & -27.3 & \pm & -24.0 & $* * *$ & -17.7 & \pm & -18.0 & & -29.5 & \pm & -29.1 & \\
\hline & 24 & -6.9 & \pm & -6.6 & & -27.2 & \pm & -23.9 & $* * *$ & -9.3 & \pm & -10.2 & & -32.1 & \pm & -31.3 & \\
\hline & 25 & 0.0 & \pm & 0.0 & & -27.6 & \pm & -24.1 & $* * *$ & 0.0 & \pm & 0.0 & & -33.0 & \pm & -32.3 & \\
\hline & 26 & 7.1 & \pm & 7.4 & & -27.3 & \pm & -24.0 & $* * *$ & 9.4 & \pm & 11.5 & * & -31.7 & \pm & -31.0 & \\
\hline & 27 & 14.4 & \pm & 14.3 & & -27.1 & \pm & -24.0 & $* * *$ & 17.1 & \pm & 18.7 & & -29.5 & \pm & -29.1 & \\
\hline & 28 & 21.7 & \pm & 21.5 & & -27.0 & \pm & -24.6 & $* *$ & 24.9 & \pm & 25.9 & & -25.3 & \pm & -25.4 & \\
\hline & 29 & -21.8 & \pm & -21.0 & & -33.2 & \pm & -30.5 & $* *$ & -26.2 & \pm & -25.4 & & -31.8 & \pm & -33.6 & \\
\hline & 30 & -14.8 & \pm & -13.8 & * & -37.7 & \pm & -34.6 & $* *$ & -17.8 & \pm & -17.9 & & -39.6 & \pm & -39.3 & \\
\hline & 31 & -7.6 & \pm & -6.6 & * & -40.3 & \pm & -36.5 & $* * *$ & -9.4 & \pm & -10.2 & & -43.6 & \pm & -42.2 & \\
\hline & 32 & 0.0 & $\begin{array}{l} \pm \\
\pm\end{array}$ & 0.0 & & -41.2 & \pm & -37.2 & $* * *$ & 0.0 & $\begin{array}{l} \pm \\
\pm\end{array}$ & 0.0 & & -45.0 & $\begin{array}{l} \pm \\
\pm\end{array}$ & -43.5 & \\
\hline & 33 & 6.9 & $\begin{array}{l} \pm \\
\pm\end{array}$ & 7.4 & & -40.4 & $\begin{array}{l} \pm \\
\pm\end{array}$ & -36.5 & $* * *$ & 9.3 & $\begin{array}{l} \pm \\
\pm\end{array}$ & 11.6 & $*$ & -43.5 & $\begin{array}{l} \pm \\
\pm\end{array}$ & -41.6 & \\
\hline & 34 & 14.3 & \pm & 14.2 & & -37.7 & \pm & -34.8 & $* *$ & 17.0 & \pm & 18.7 & $*$ & -39.9 & \pm & -38.6 & \\
\hline & 35 & 21.6 & \pm & 21.6 & & -32.9 & \pm & -31.1 & $*$ & 24.9 & \pm & 25.9 & & -32.4 & \pm & -33.1 & \\
\hline
\end{tabular}

* Indicates significant difference in the Class II pretreatment group from the Class III pretreatment group. * $\mathrm{P}<0.05 ; * * \mathrm{P}<0.01$; ${ }^{* * *}<0.001$.

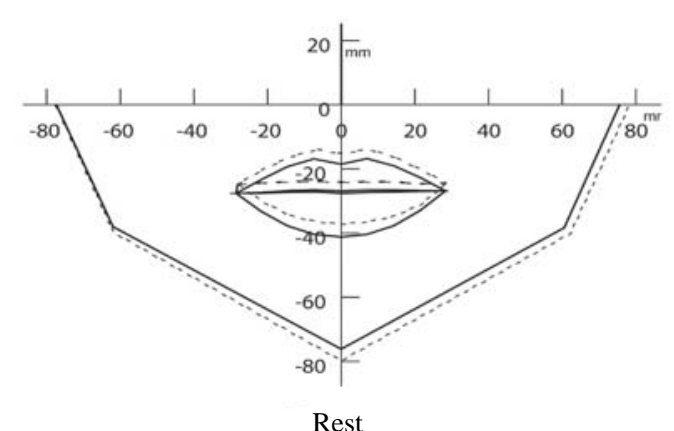

Figure 2. Graphics of mean value of landmarks for the pretreatment class II group (black) and class III group (dotted).
10

Bangladesh Journal of Orthodontics and Dentofacial Orthopedics (BJO \& DFO) 
Lip Morphological Changes and Differences before and after Orthodontic Treatment.

Table 7. Comparism between the Class II posttreatment group from the Class III posttreatment group

\begin{tabular}{|c|c|c|c|c|c|c|c|c|c|c|c|c|c|c|c|c|}
\hline \multirow{3}{*}{ Point } & & \multicolumn{7}{|c|}{ Post Rest } & \multicolumn{8}{|c|}{ Post Smile } \\
\hline & & \multicolumn{3}{|c|}{$\mathrm{X}$} & \multicolumn{4}{|c|}{$\mathrm{Y}$} & \multicolumn{4}{|c|}{$\mathrm{X}$} & \multicolumn{4}{|c|}{$\mathrm{Y}$} \\
\hline & & $\begin{array}{l}\text { ClassII } \\
\text { Mean }\end{array}$ & \pm & $\begin{array}{l}\text { ClassIII } \\
\text { Mean } \\
\end{array}$ & $\begin{array}{l}\text { ClassII } \\
\text { Mean }\end{array}$ & \pm & $\begin{array}{l}\text { ClassII } \\
\text { Mean }\end{array}$ & & $\begin{array}{l}\text { ClassII } \\
\text { Mean }\end{array}$ & \pm & $\begin{array}{l}\text { ClassII } \\
\text { Mean } \\
\end{array}$ & & $\begin{array}{l}\text { ClassII } \\
\text { Mean }\end{array}$ & \pm & $\begin{array}{l}\text { ClassIII } \\
\text { Mean }\end{array}$ & \\
\hline \multirow[t]{5}{*}{ Outline, mm } & 1 & -75.3 & \pm & -76.9 & 0.0 & \pm & 0.2 & & -77.3 & \pm & -78.9 & & 0.0 & \pm & 0.1 & \\
\hline & 2 & -60.2 & \pm & -61.3 & -38.9 & \pm & -38.7 & & -61.7 & \pm & -62.8 & & -40.2 & \pm & -40.4 & \\
\hline & 3 & 0.9 & \pm & 0.0 & -77.9 & \pm & -77.7 & & 0.2 & \pm & 0.0 & & -80.7 & \pm & -80.7 & \\
\hline & 4 & 61.6 & \pm & 61.4 & -38.8 & \pm & -38.9 & & 62.0 & \pm & 63.4 & & -40.4 & \pm & -40.5 & \\
\hline & 5 & 76.1 & \pm & 76.9 & 0.0 & \pm & -0.1 & & 77.2 & \pm & 79.5 & * & 0.0 & \pm & 0.1 & \\
\hline \multirow[t]{16}{*}{ Upper lip, mm } & 6 & -29.1 & \pm & -29.3 & -26.7 & \pm & -24.8 & & -35.9 & \pm & -36.4 & & -20.0 & \pm & -16.6 & $* *$ \\
\hline & 7 & -22.2 & \pm & -21.7 & -23.4 & \pm & -20.5 & $* * *$ & -27.4 & \pm & -27.2 & & -16.4 & \pm & -13.8 & $* *$ \\
\hline & 8 & -14.8 & \pm & -14.3 & -19.9 & \pm & -17.0 & $* * *$ & -19.0 & \pm & -19.0 & & -14.5 & \pm & -11.9 & $* *$ \\
\hline & 9 & -7.5 & \pm & -7.1 & -17.5 & \pm & -14.9 & $* * *$ & -10.5 & \pm & -10.7 & & -13.5 & \pm & -10.8 & $* *$ \\
\hline & 10 & 0.0 & \pm & 0.0 & -19.2 & \pm & -16.0 & $* * *$ & 0.0 & \pm & 0.0 & & -14.7 & \pm & -11.8 & $* * *$ \\
\hline & 11 & 7.2 & \pm & 7.4 & -17.4 & \pm & -14.7 & $* * *$ & 10.6 & \pm & 12.9 & * & -13.5 & \pm & -10.6 & $* * *$ \\
\hline & 12 & 14.8 & \pm & 14.5 & -19.5 & \pm & -16.8 & $* * *$ & 19.0 & \pm & 20.6 & * & -14.5 & \pm & -11.8 & $* *$ \\
\hline & 13 & 22.6 & \pm & 21.9 & -23.0 & \pm & -20.6 & $* *$ & 27.1 & \pm & 28.3 & & -16.4 & \pm & -13.8 & $* *$ \\
\hline & 14 & 29.3 & \pm & 29.3 & -26.2 & \pm & -24.2 & $* *$ & 35.4 & \pm & 36.3 & & -19.6 & \pm & -16.0 & $* *$ \\
\hline & 15 & -22.2 & \pm & -21.7 & -26.6 & \pm & -24.7 & * & -27.4 & \pm & -27.2 & & -18.9 & \pm & -16.7 & * \\
\hline & 16 & -14.9 & \pm & -14.4 & -26.2 & \pm & -24.6 & $*$ & -18.9 & \pm & -19.0 & & -18.6 & \pm & -16.7 & * \\
\hline & 17 & -7.5 & \pm & -7.0 & -26.5 & \pm & -24.7 & $* *$ & -10.4 & \pm & -10.7 & & -18.9 & \pm & -16.7 & $*$ \\
\hline & 18 & 0.0 & \pm & 0.0 & -27.0 & \pm & -24.9 & $* *$ & 0.0 & \pm & 0.0 & & -20.3 & \pm & -17.5 & $* *$ \\
\hline & 19 & 7.2 & \pm & 7.3 & -26.6 & \pm & -24.7 & $* *$ & 10.6 & \pm & 12.9 & $*$ & -19.1 & \pm & -16.8 & $* *$ \\
\hline & 20 & 14.8 & \pm & 14.5 & -26.3 & \pm & -24.6 & $* *$ & 18.9 & \pm & 20.6 & $*$ & -18.6 & \pm & -16.7 & $*$ \\
\hline & 21 & 22.5 & \pm & 22.0 & -26.4 & \pm & -24.7 & $*$ & 27.1 & \pm & 28.2 & & -18.7 & \pm & -16.8 & \\
\hline \multirow[t]{14}{*}{ Lower lip, mm } & 22 & -22.2 & \pm & -21.7 & -26.7 & \pm & -24.8 & $*$ & -27.5 & \pm & -27.1 & & -25.8 & \pm & -24.1 & $*$ \\
\hline & 23 & -14.9 & \pm & -14.4 & -26.4 & \pm & -24.8 & $*$ & -19.0 & \pm & -19.0 & & -30.4 & \pm & -28.8 & \\
\hline & 24 & -7.6 & \pm & -7.0 & -26.8 & \pm & -24.8 & $* *$ & -10.5 & \pm & -10.7 & & -33.4 & \pm & -31.3 & * \\
\hline & 25 & 0.0 & \pm & 0.0 & -27.2 & \pm & -25.0 & $* *$ & 0.0 & \pm & 0.0 & & -34.4 & \pm & -32.4 & * \\
\hline & 26 & 7.2 & \pm & 7.3 & -26.9 & \pm & -24.8 & $* *$ & 10.5 & \pm & 13.0 & $* *$ & -33.3 & \pm & -30.7 & $*$ \\
\hline & 27 & 14.8 & \pm & 14.5 & -26.5 & \pm & -24.8 & $*$ & 19.0 & \pm & 20.6 & $*$ & -30.3 & \pm & -28.4 & \\
\hline & 28 & 22.4 & \pm & 22.0 & -26.5 & \pm & -24.8 & $*$ & 27.3 & \pm & 28.3 & & -25.7 & \pm & -23.9 & \\
\hline & 29 & -22.2 & \pm & -21.7 & -32.1 & \pm & -31.2 & & -27.5 & \pm & -27.2 & & -32.5 & \pm & -31.8 & \\
\hline & 30 & -14.8 & \pm & -14.4 & -37.3 & \pm & -35.4 & $* *$ & -19.1 & \pm & -19.0 & & -40.4 & \pm & -38.3 & \\
\hline & 31 & -7.5 & \pm & -7.2 & -40.0 & \pm & -37.5 & $* * *$ & -10.5 & \pm & -10.6 & & -44.7 & \pm & -42.0 & * \\
\hline & 32 & 0.0 & \pm & 0.0 & -40.8 & \pm & -38.2 & $* * *$ & 0.0 & \pm & 0.0 & & -46.3 & \pm & -43.8 & * \\
\hline & 33 & 7.2 & \pm & 7.3 & -40.0 & \pm & -37.5 & $* *$ & 10.5 & \pm & 13.0 & * & -44.8 & \pm & -41.0 & $* *$ \\
\hline & 34 & 14.9 & \pm & 14.4 & -37.2 & \pm & -35.5 & $*$ & 19.0 & \pm & 20.6 & $*$ & -40.8 & \pm & -37.4 & $* *$ \\
\hline & 35 & 22.6 & \pm & 22.0 & -32.1 & \pm & -31.2 & & 27.2 & \pm & 28.3 & & -33.1 & \pm & -30.8 & \\
\hline
\end{tabular}

* Indicates significant difference in the Class II posttreatment group from the Class III posttreatment group. * $\mathrm{P}<0.05$; ** $<<0.01 ; * * * \mathrm{P}<0.001$
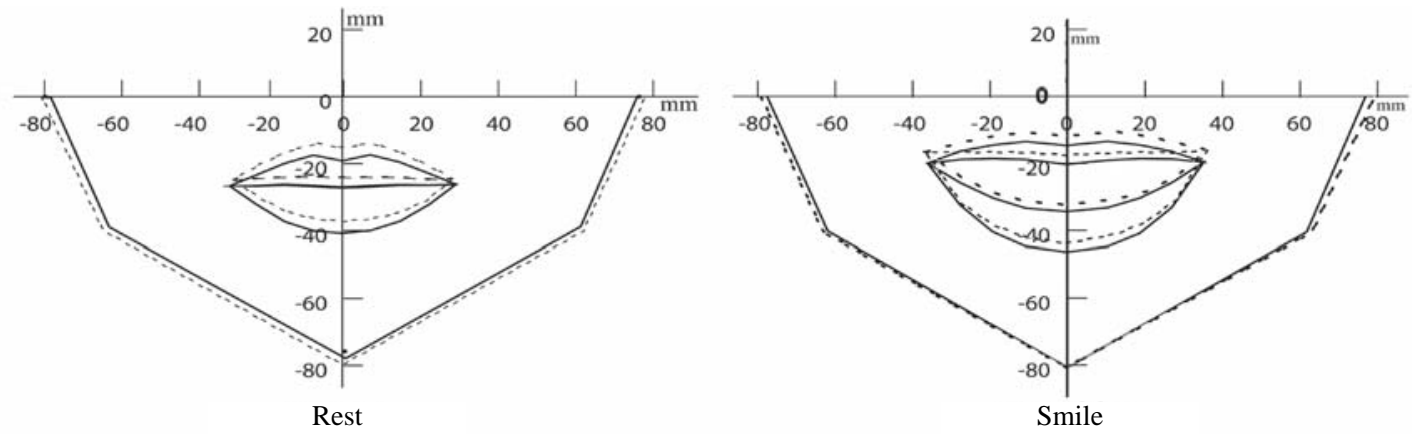

Figure 3. Graphics of mean value of landmarks for the posttreatment class II (black) and class III group (dotted).

Bangladesh Journal of Orthodontics and Dentofacial Orthopedics (BJO \& DFO) 


\section{DISCUSSION}

The facial esthetic is one of the important social concerns in current society. Eighty percent of patients seek ${ }^{12}$ orthodontic treatment for esthetic reasons. Therefore, orthodontic treatment has gained momentum in modern society, and therefore, will attract even more attention in the future. The success of orthodontic treatment is routinely assessed by smile esthetics, and the lips are the controlling factor in the smile.

The advantage of this facial photograph based study is that the procedure is simple and economical, and the number of samples is easily increased. In addition, these photographs are usually available in the orthodontic office, and they are rated as more attractive than the profile views. However, the present study revealed that there were significant differences of the coordinates of landmarks at rest and on smiling between the Class II and Class III patient group., and they were similar to the result in the previous study $11,13-15$.

\section{CONCLUSIONS}

The hypothesis is true that there is a different in the morphology of the lips of Class II div 1 and Class III malocclusion at rest and on smiling conditions, before and after orthodontic treatment. Orthodontic treatment induced larger amount in movement of lips at rest and on smiling in both class II and class III patients, which may reflect the emotional changes in the patients after treatment. The findings of this study may therefore be valuable for future research on the morphological changes of soft tissue in the lip area after retention.

\section{REFERENCES}

1. Subtelny JD. The soft tissue profile, growth and treatment changes. Angle Orthod. 1961; 31:105-122.

2. Wylie WL. The Mandibular Incisor- Its Role in Facial Esthetics. Angle Orthod. 1955; 25:32-41.

3. Zachrisson BU. Esthetic factors involved in anterior tooth display and the smile: vertical dimension. J Clin Orthod. 1998;32:432-445

4. Roy Sabri. The Eight Components of a Balanced Smile. J Clin Orthod. 2005;39(3): 155-167.
5. Wylie WL. The Mandibular Incisor- Its Role in Facial Esthetics. Angle Orthod. 1955; 25:32-41.

6. Cummins DM, Bishara SE, Jakobsen JR. A computer assisted photogrammetric analysis of soft tissue changes after orthodontic treatment. Part II: Results. Am J Orthod Dentofacial Orthop. 1995;108:38-47.

7. Foster EJ. Profile preference among diversified groups. Angle Orthod. 1973; 43:34-40.

8. Mackley RJ. An evaluation of smiles before and after orthodontic treatment. Angle Orthod. 1993;63:183-190.

9. Kerr W J S, O`Donnel J M. Panel perception of facial attractiveness. Brit J Orthod. 1990; 17:299-304.

10. Sarver, D.M.: The importance of incisor positioning in the esthetic smile: The smile arc, Am. J. Orthod. 120:98-111, 2001.

11. Islam R, Kitahara T, Naher L, Hara A, Nakasima A. Lip morphological changes in orthodontic treatment: class II division 1 Malocclusion and normal occlusion at rest and on smiling. Angle Orthod. 2009;79(2):256-264.

12. Albino JE, Cunat JJ, Fox RN, Lewis EA, Slakter MJ, Tedesco LA. Variables discriminating individuals who seek orthodontic treatment. J Dent Res. 1981;60:1661-1667.

13. Ishikawa T, Saito Y, Muraoka S, Kitahara T, Ioi H, Nakasima A. Three-dimensional analysis of smile movement using high speed cameras for the subjects with normal occlusions and jaw deformities. Orthodontic Waves-Jpn Ed. 2007;66: 92-105.

14. Islam R, Kitahara T, Naher L, Hara A, Nakasima A. Lip morphological changes in orthodontic treatment: class II division 1 Malocclusion and normal occlusion at rest and on smiling. Angle Orthod. 2009;79(2):256-264.

15. Rafiqul Islam1, Toru Kitahara2, Lutfun Naher3, Atsushi Hara4, and Shunsuke Nakata5 Lip Morphological Changes following Orthognathic Surgery for Class III Malocclusion. Angle Orthod. 2010;80 (2):344-353.

\section{Correspondence}

Dr Md. Rafiqul Islam, BDS, PhD

Department of Orthodontics, Pioneer Dental College, Dhaka University, Bangladesh.

This study was conducted in the Department of Orthodontics, Graduate School of Dentistry, Kyushu University, Fukuoka, Japan.

E-mail: rafiqdr_007@yahoo.com, Cell: +81-1749-881-576 